\title{
Biologic Therapy in Uveitis
}

\author{
Simon R Taylor
}

University of Surrey and Royal Surrey County Hospital, Surrey, UK

DOI: http://doi.org/10.17925/EOR.2016.10.01.17

\begin{abstract}
The treatment of uveitis is undergoing significant change as a result of the development of new therapeutic approaches, of which the biologic agents form a major part. These targeted therapies have shown great promise for the treatment of refractory disease and some have now undergone systematic evaluation through prospective clinical trials, unlike many of their predecessor drugs.
\end{abstract}

\section{Keywords}

Uveitis, biologic agents, adalimumab, infliximab

\begin{abstract}
Disclosure: Simon R Taylor has participated in advisory boards for AbbVie, Alimera, Novartis and Santen, received conference attendance and travel grants from Alimera, Allergan and Novartis and received speaker fees from Allergan and Novartis. No funding was received in the publication of this article. This article is a short opinion piece and has not been submitted to external peer reviewers.

Open Access: This article is published under the Creative Commons Attribution Noncommercial License, which permits any noncommercial use, distribution, adaptation, and reproduction provided the original author(S) and source are given appropriate credit.

Received: 17 July 2016 Published Online: 4 August 2016 Citation: European Ophthalmic Review, 2016;10(1):17-8

Correspondence: Simon R Taylor, Department of Ophthalmology, Royal Surrey County Hospital, Egerton Road, Guildford GU2 7XX, UK. E: s.r.taylor@surrey.ac.uk
\end{abstract}

This last decade has seen truly significant changes in the understanding and management of uveitis, from the standardisation of diagnostic criteria that the Standardization of Uveitis Nomenclature (SUN) project has brought to the advent of novel therapeutic options that have revolutionised disease management. ${ }^{1,2}$ In this, the uveitis world is following the lead set by rheumatology in the 1980s, in which increasingly scientific disease phenotyping drove better and more ambitious clinical trials that in turn drove improvements in clinical management and outcomes. This is making it an exciting time to be part of uveitis research, and the increasing number of well-designed clinical trials is testament to this.

The need for novel treatment options lies in the side-effect profile of corticosteroids. Their ease of use and reliability means that they remain the mainstay of initial treatment, particularly when inflammation is bilateral or associated with systemic disease, but their side-effect profile is significant. Conventional steroid-sparing immunosuppressive agents are of variable efficacy and carry their own issues with sideeffects, driving the development of two alternative approaches.

The first of these is the increasing use of local therapy in the form of sustained release steroid implants such as the dexamethasone implant (Ozurdex ${ }^{\circledR}$; Allergan, Inc., Irvine, CA, US) and fluocinolone implant (Retisert ${ }^{\mathrm{TM}}$; Bausch \& Lomb Inc., Rochester, NY, US). ${ }^{3,4}$ These have been tested in clinical trials programmes and are very effective in some patients, particularly in the absence of systemic disease or for unilateral disease. However, they are all currently corticosteroidbased and thus have associated ocular side-effects such as cataract formation and raised intraocular pressure.

The second approach is the development of more targeted systemic therapies, namely the biologic agents. In contrast to conventional immunosuppressive agents, biologics modulate inflammation by targeting specific receptors or single molecules and thus offer a more specific suppression of the immune effector response. They are useful in patients who fail to respond to conventional immunosuppressive therapy, ${ }^{5}$ and may also reduce the risk of visual loss in severe forms of intraocular inflammation, such as uveitis related to Behcet's disease. ${ }^{6}$

The greatest volume of evidence for the biological agents exists for the tumour necrosis factor (TNF)-alpha inhibitors. TNF- $\alpha$ has been detected in human eyes with a variety of inflammatory conditions and its inhibition can be effective in the short and long-term management of patients with refractory posterior uveitis. ${ }^{7}$ The most established drug in this class is infliximab (Remicade ${ }^{\circledR}$; Janssen Biotech, Inc., Horsham, PA, US), but the first biologic drug to undergo a formal clinical trial programme in uveitis was adalimumab (Humira ${ }^{\circledR}$; AbbVie Inc. North Chicago, IL, US). The development of clinical trials is important, as it enables these drugs to move from off-label and ad hoc use to formal licensing and national commissioning. Humira is a fully humanised monoclonal antibody directed against soluble and membrane-bound TNF which has the advantage of being a subcutaneous injection that patients can perform at home, rather than requiring hospital admission for intravenous infusions. ${ }^{8}$ Humira was approved by the US Food and Drug Administration (FDA) earlier this year, and an application for EU marketing authorisation is expected shortly. The drug is also due to be part of a UK National Institute for Health and Care Excellence (NICE) multiple technology appraisal.

Other biologic agents provide interest, but are at an earlier stage of understanding and usage. Interleukin-17 (IL-17) has generated a great deal of interest as a possible therapeutic target, but secukinumab, a human monoclonal antibody directed against IL-17, failed to demonstrate efficacy in early trials in uveitis; 9 nevertheless, this approach holds out the hope of interrupting a mechanism that may be involved in the generation of tolerance. ${ }^{10}$ Other novel systemic 


\section{Editorial Uveitis}

approaches include reducing antigen presentation by blocking lymph node efflux via the sphingosine-1-phosphate receptor (fingolimod) targeting B cells (rituximab), and reducing inflammatory cell migration via $\alpha 4$-integrin (natalizumab).
It is an exciting time in uveitis research - there are more therapeutic options than ever before, yet there are also more questions about how and when to use them; the future should hold interesting developments for researchers, clinicians and patients.
1. Jabs DA, Nussenblatt RB, and Rosenbaum JT, Standardization of uveitis nomenclature for reporting clinical data. Results of the First International Workshop, Am I Ophthalmol, 2005;140:509-16

2. de Smet MD, Taylor SR, Bodaghi B, et al., Understanding uveitis: the impact of research on visual outcomes, Prog Retin Eye Res, 2011:30:452-70

3. JH Kempen JH, Altaweel MM, Holbrook JT, et al., Randomized comparison of systemic anti-inflammatory therapy versus fluocinolone acetonide implant for intermediate, posterior and panuveitis: the multicenter uveitis steroid treatment trial,
Ophthalmology, 2011;118:1916-26.

4. Lowder C, Belfort R Jr, Lightman S, et al., Dexamethasone intravitreal implant for noninfectious intermediate or posterior uveitis, Arch Ophtha/mol 2011:129:545-53.

5. Joshi L, Lightman SL, Salama AD, et al., Rituximab in refractory ophi, Light Wen ophthalmic wegener's granulom, predict relapse, but repeat treatment can be effective, Ophthalmology, 2011,118.2498-503.

Behcet disease: visual prognosis and factors influencing the development of visua loss, Am J Ophthalmol, 2011;152:1059-66.
7. Benitez-del-Castillo JM, Martinez-de-la-casa JM, et al, Long-term treatment of refractory posterior uveitis with antiTNFalpha (infliximab), Eye (Lond), 2005;19:841-5.

8. Diaz-Llopis M, Salom D, Garcia-de-Vicuna C, et al., Treatment of refractory uveitis with adalimumab: a prospective multicenter study of 131 patients, Ophthalmology, 2012:119:1575-81.

9. Dick AD, Tugal-Tutkam, Foster $S$, et al., Secukinumab in the Dick AD, Tugal-Tutkam, Foster S, et al., Secukinumab in the
treatment of noninfectious uveitis: results of three randomized, controlled clinical trials, Ophthalmology, 2013;120:777-87.

10. Barbi J, Pardoll D, Pan F, Metabolic control of the Treg/Th17 axis, Immunol Rev, 2013;252:52-77. 